\title{
SPIN CORRELATIONS IN QUARK AND GLUON FRAGMENTATION
}

\author{
Martin B. Einhorn
}

Randall Lab of Physics, University of Michigan, Ann Arbor, MI 48109

\section{ABSTRACT}

The possibilities for spin correlations in quark and gluon fragmentation are assessed. It is pointed out that heavy quarks, especially charm, offer substantial potential and that there is currently almost no experimental evidence one way or the other concerning the magnitude of such correlations. The $Q^{2}$-evolution of fragmentation functions is discussed briefly, emphasizing in particular that the mean quark and gluon fragmentation asymmetries grow as $\ln Q^{2}$.

\section{INTRODUCTION}

It is well known that, in QCD, the probability of finding a quark $q_{ \pm}$of helicity $\pm 1 / 2$ in a polarized hadron $h$ of helicity $\lambda$ is described by a structure function $q_{ \pm}^{h_{\lambda}}\left(x ; Q^{2}\right)$, where $x$ is the longitudinal momentum fraction of the hadron carried by the quark and $Q$ is the momentum scale associated with the "resolution" of the process under consideration. ${ }^{\star 1,2]}$ The dependence on $x$ is nonperturbative and cannot now be calculated reliably, whereas the dependence on $Q^{2}$ can be calculated perturbatively. Analagously, when a quark $q$ of definite helicity fragments into a jet of hadrons, the probability of finding a hadron $h$ of helicity $\lambda$ is described by a so-called fragmentation function $D_{q_{ \pm}}^{h_{\lambda}}\left(z ; Q^{2}\right){ }^{[3]}$ Here, $z$ is the longitudinal momentum fraction of the quark carried by the hadron. Again, the $z$ dependence is not perturbatively calculable, but the $Q^{2}$ dependence is. Similarly, one defines $D_{G_{ \pm}}^{h_{\lambda}}$ for the fragmentation function for a gluon $G_{ \pm}$of helicity \pm 1 . While there exists data on various unpolarized fragmentation functions, there is but scant information on the helicity correlations in fragmentation. In this talk, I want to talk briefly about two distinct aspects of spin correlations in quark and gluon fragmentation: (1) The nonperturbative $z$-dependence. If there were strong spin correlations for heavy quarks, the $c, b$, or $t$ quarks, this could be a very important tool in experimental analyses. We will review what little is known, emphasizing that in principle these correlations could be very large and as yet unobserved. (2) The perturbative $Q^{2}$-dependence. Here we shall review some results derived about three years ago, ${ }^{[4]}$ the details of which were reported in last week's Theory Workshop and which will be published elsewhere. ${ }^{(5)}$ We shall show that, as with the gluonic asymmetries in structure functions ${ }^{[6-9]}$ there are some rather surprising, nonintuitive results in store for spin asymmetries in quark and gluon fragmentation. ${ }^{\dagger}$

* The transverse momentum $p_{\perp}$ has been ignored (i.e.,summed over.)

† At the time we wrote Ref. 9, I was unaware of the work of Refs. 6 and 8, so let me take this occasion to acknowledge their pioneering work, which also contained the germs of recent discussions about the role of the anomaly in the axial current. 


\section{SPIN CORRELATIONS IN HEAVY QUARK FRAGMENTATION}

Taking up the nonperturbative issues first, it is well known experimentally and rather well understood phenomenologically in the so-called Lund model of fragmentation ${ }^{[10]}$ that in the process of "hadronization," very few heavy quarks are created, in contract to the $u, d, s$ quarks. Thus, when a gluon fragments, there will only be glueballs or mesons and baryons containing light $(u, d, s)$ quarks in the final state, in contrast to the "flavor independence" of the gauge coupling of gluons to quarks. When a $c, b$, or $t$-quark fragments, to a high degree of accuracy, there is only a single hadron in the resulting jet carrying the $c, b$,ort-flavor, respectively. Thus, if there is a spin correlation between, say, the charmed quark and the resulting charmed hadron, there is no dilution due to a multiplicity of charmed hadrons in the final state, in marked contrast with an attempt to discern such a correlation in the production of $\rho, \omega$, or $\phi$ mesons for example. ${ }^{*}$ Our interest is in the fragmentation of heavy quarks with energies large compared to their mass so that there is an approximately scaling fragmentation probability $D\left(z ; Q^{2}\right)$ and mass effects can be less ignored in determining the $Q^{2}$-dependence. From an experimental standpoint, charmed quarks are a particularly good example, since it is easy to produce them fast $\left(m_{c} \approx 1.2 \mathrm{GeV}\right)$, and fast charmed hadrons can be efficiently produced and detected both in $e^{-} e^{+}$annihilation and by hadronic collisions. One would anticipate on general grounds and it is observed that, when a $c$-quark fragments, it produces mostly $D$ and $D^{*}$ mesons, with occasional $D^{* *}$ mesons and rarely (but detectably) baryons.

The first question is what is the relative abundance of $D$ 's and $D^{*}$ 's. Since there mass is so close, one might expect on statistical grounds that there would be a $3: 1$ ratio of $D^{*}$ to $D$, and indeed, this is consistent with observations. The most precise experimental result is quoted by CLEO, with the probability of a $c$-quark fragmenting to a $D^{*}$ equal to $P_{V}=0.85 \pm 0.11 \pm 0.17 .^{[11]}$ Similar results are quoted by HRS at PEP ${ }^{[12]}$ and ARGUS at DORIS. ${ }^{[13]}$ Further information comes from an HRS analysis ${ }^{[14]}$ of the alignment in $e^{-} e^{+} \rightarrow D^{*} X$ at $29 \mathrm{GeV}$. The alignment is defined in terms of the spin density matrix of the $D^{*}$ as $\eta \equiv 1 / 2\left(2 \rho_{00}-\rho_{11}-\rho_{-1-1}\right)$. By parity conservation and the normalization of the density matrix, this can be simplified to $\eta=1 / 2\left(3 \rho_{00}-1\right)$. With a negligible parity non-conservation in the production at this energy, one in principle obtains equal numbers of left- and right-handed $c$-quarks, so the best one could hope for is to distinguish transverse from longitudinal $D^{*}$ production. From an analysis of the $D \pi$ angular distribution, a determination was made of $\rho_{0,0}$, and it was found to be approximately $1 / 3$. (In addition, the

* Of course, a c-quark is eventually produced by weak decays of B-mesons, so if both $c$ and $b$ are produced initially, we cannot know immediately that a charmed hadron in the final state is a $c$-quark fragment and a $b$-quark decay product. Similar remarks presumably apply to the $t$-quark, but the $t$ is apparently so much heavier than either the $c$ or the $b$ that the degree of contamination is likely to be insignificant. 
non-diagonal elements $\rho_{1,-1}$ and $\Re e \rho_{1,0}$ are small, as required for a parton-like description of the process.) The natural inference from this is that the 3 spin states of the $D^{*}$ are equally populated and for unpolarized initial $c$-quark, the evidence is consistent with this expectation.

The preceding facts have led many to assume that there will be no interesting spin correlations in $c$-quark fragmentation, but this is not necessarily the case, as reference to a simple model will illustrate: Consider, say, a right-handed $c$-quark and suppose it hadronizes by picking up a light quark forming either a peudoscalar $\mathrm{P}\left(D^{0}, D^{+}\right.$, or $\left.F\right)$ or their vector counterparts $\mathrm{V}\left(D^{0^{*}}, D^{+^{*}}\right.$, or $F^{*}$.) Suppose the forces are entirely spin independent so that (a) the $c$-quark helicity remains entirely undisturbed by the hadronization process and (b) the light quark which is picked up has equal probability to have either parallel or opposite helicity. If it is parallel, then of course you have a vector meson $\mathrm{V}$ with helicity +1 . If it is opposite, then you have equal probability of forming a longitudinal vector $V$ or a pseudoscalar $P$. You obviously never can form a $V$ with helicity -1 . By parity conservation, this also determines the fragmentation of a $c$-quark with helicity $-1 / 2$. Thus, this random pickup model leads to the predictions of Table 1.

Table I Alternate models of helicity correlations

\section{Uncorrelated}

\begin{tabular}{cc|ccc} 
& $c_{ \pm}$ & $c_{+}$ & $c_{-}$ & Mean \\
$D_{+1}^{*}$ & $1 / 4$ & $1 / 2$ & 0 & $1 / 4$ \\
$D_{0}^{*}$ & $1 / 4$ & $1 / 4$ & $1 / 4$ & $1 / 4$ \\
$D_{-1}^{*}$ & $1 / 4$ & 0 & $1 / 2$ & $1 / 4$ \\
$D$ & $1 / 4$ & $1 / 4$ & $1 / 4$ & $1 / 4$
\end{tabular}

\section{$\underline{\text { Random Pickup }}$}

Mean

$1 / 4$

$1 / 4$

$1 / 4$

$1 / 4$

Thus, for a right-handed c-quark, there is a $50 \%$ probability that the charmed hadron in the jet is a right-handed $\mathrm{V}$. This is obviously a very strong spin correlation, one which is large enough to be experimentally useful. Yet in unpolarized production and detection, in which one sums over the left and righthanded $c$-quark, as the last column in Table 1 shows, this it random pickup model leads to predictions identical to a completely uncorrelated, statistical model in which each spin state is equally populated. I want to emphasize that there is no evidence at this time to distinguish the statistical model from the random pickup model or any of an infinite number of other models with the same property for unpolarized experiments.

How could one determine these spin-correlated fragmentation functions ex- 
perimentally? This has not been thoroughly investigated, but a couple of methods come to mind: One obvious way would be to arrange to have $c$-quarks of a definite helicity. Since the charged weak current is left-handed, looking for a $D^{*}$ in a charmed quark jet in a semileptonic weak decay or in deeply inelastic lepton scattering is attractive. The process $B \rightarrow D^{*} X e \nu$ could potentially manifest $D^{*}$ polarization. However, the energy of the charmed quark arising in this decay is not very large with respect to its mass, so this is unlikely to be useful.

As for deeply inelastic scattering, because of the separation of the struck quark jet from the target fragments, HERA will probably be the best place to study the fragmentation of the struck quark via $e^{+} p \rightarrow \bar{\nu}_{e} D^{*} X$. The analysis of the $D^{*} \rightarrow D \pi$ angular distribution, in particular, or the asimuthal angle of the $D \pi$ decay plane, will enable one to deduce the various fragmentation functions. This will require substantial numbers of events, but, for these purposes, the charged weak current is simply a device for selecting the $c$-quark of definite helicity, and there is no need to go to very high $Q^{2}$. A second method that occurs is to exploit parity violation in $Z^{0}$ decay, but it is well-known ${ }^{[15]}$ that the preference for a particular helicity in $Z^{0} \rightarrow q \bar{q}$ is small, because it happens that $1-4 \sin ^{2} \theta_{w}$ is small. Nevertheless, with the helicity conserving vector or axial vector coupling, there is a perfect correlation between the helicities of the produced $c \bar{c}$-pair, viz., if the $c$-quark is left-handed, the $\bar{c}$ will be righthanded, and vice-versa. Based on the aforementioned observations by CLEO, HRS, and ARGUS, at least half of the events will have a $D^{*} \overline{D^{*}}$ pair in the final state. Since one anticipates at SLC and LEP very large numbers of events, despite reconstruction efficiencies, one may be able to examine the correlations in the $D^{*}$ and $\overline{D^{*}}$ decay distributions for helicity information. ${ }^{*}$ Furthermore, if longitudinally polarized $e^{-}$beams are available, the analysis may be broken down further since the final state arises from the decay of a unique $Z^{0}$ helicity state.

One may also look for helicity correlations in rare but distinctive fragmentation such as $c \rightarrow \Lambda_{c}$ and $c \rightarrow \psi / J$. Given the rate of $c$-quark production at high energies and the continued refinement of vertex detectors and triggering techniques, this may not be as far-fetched as it may now sound.

All these observations concerning potential helicity correlations in the fragmentation of charmed quarks apply equally to fast $b$ - or $t$-quarks. However, the decay of the $B^{*}$ is more complex. Since $B^{*} \rightarrow B \pi$ is kinematically forbidden, one presumably must rely on an analysis of the $B \gamma$ decay mode. Our thoughts about the origin of polarized $b$-quarks are analogous to our remarks

* In comments following my talk, G. R. Goldstein alluded to recent work by him, Dalitz, and Marshall ${ }^{[16]}$ in which they independently made this observation concerning the utility of $D^{*}-\overline{D^{*}}$ helicity correlations. (See Goldstein's summary in these Proceedings.) I wish to thank him for for providing me with preliminary drafts of the papers cited in Ref. 16. 
on $c$-quarks. Thus, $B^{*}$ production in polarized $e^{-} e^{+}$annihilation or $Z$-decay is one possibility. Depending on how heavy the top quark turns out to e, the semileptonic decays $T \rightarrow B^{*} X \ell \nu$ may be promising. I have not thought about whether in practice or nonleptonic weak decays such as $B^{*} \rightarrow D \pi$ or $B^{*} \rightarrow D^{*} \pi$ could be used for this purpose. The $t$ may be so heavy that it would be very rare that its mass could be neglected, even at the SSC. In addition, vertex detection is only possible with charmed mesons, so charm is by far the most attractive candidate for these studies.

Unless, it can be measured, there isn't much point in talking about spin correlations in gluon fragmentation. I haven't spent much time thinking about this, and this is a subject for further research. However, although not a first generation experiment, in the course of time, it may become something to consider. For example, three-jet $(q q G)$ production in $e^{-} e^{+}$annihilation has been discussed in this context. ${ }^{[17]}$ For unpolarized beams, there is a small polarization (10\%-50\%, depending on direction) of the final state induced by parity violation at the $Z^{0}$. For polarized electrons, the polarization transferred to the charm quark approaches 1 , and there is a substantial gluon polarization as well. To use such an approach, one needs to be able to distinguish $c$ - or possibly $b$-quarks from gluon jets. Then one needs to be able to study the fragmentation of polarized gluons into vector mesons ${ }^{\dagger}$ or baryons in order to establish a spin correlation.

Another possibility would emerge if it turns out that there is a substantial gluon polarization in protons, a possibility much discussed at this conference in connection with the interpretation of the EMC data, ${ }^{[18]}$ If so, then, since the dominant contribution to the production of two jets in the scattering of polarized $p p$ or $\bar{p} p$ scattering is the elastic scattering of gluon pairs, it becomes a question of whether helicity information is transferred efficiently. In fact, the answer is yes. ${ }^{[19]}$ There are experiments planned for polarized beams on polarized targets at Fermilab. ${ }^{[20]}$ While not collider energies, it might be a start.

Finally, if direct studies prove impossible but there turns out to be a substantial spin correlation in quark fragmentation, then one might draw indirect information on the spin correlations in gluon fragmentation from fits to observations of the $Q^{2}$-evolution of $D_{ \pm}^{h_{\lambda}}$ for the quark, as discussed in the next Section.

In summary, it is possible that in the fragmentation of heavy quarks to hadrons with spin, especially to vector bosons, a substantial correlation persists between the quark helicity and the hadron's helicity. As fragmentation is a complex phenomenon, only experiment can tell us how much or how little. If it were substantial, this would provide a marvelous tool for experimental analyses of reactions at high energies, whether in studying weak decays of the

$\dagger$ What we wouldn't give for a readily identifiable spinning glueball! 
$b$ or $t$ or as an aid to unravelling new phenomena beyond the Standard Model. These investigations can be enhanced with polarized beams or targets, but the information can in principle be obtained using unpolarized experiments involving the charged weak current or by examining correlations between the production of pairs of vector bosons in $e^{-} e^{+}$annihilation.

\section{PREDICTIONS FOR $Q^{2}$ EVOLUTION FROM PERTURBATIVE QCD}

In principle, the predictions of perturbative $\mathrm{QCD}$ for spin correlations were contained in the original papers. ${ }^{\left[{ }^{[1]}\right]}$ However, to the best of my knowledge, they were only systematically investigated about three years ago. ${ }^{[4]}$ Here, we will only summarize these results: ${ }^{[5]}$ First of all, we need some definitions. We defined above the fragmentations functions $D_{q_{ \pm}}^{h_{\lambda}}\left(z ; Q^{2}\right)$ and $D_{G_{ \pm}}^{h_{\lambda}}\left(z ; Q^{2}\right)$ for polarized quarks and gluons, respectively. Just as for structure functions, their variation

$G$
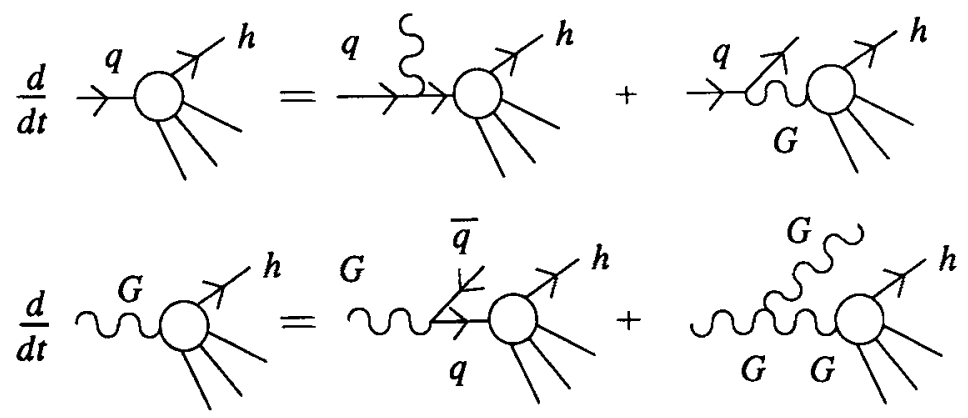

Fig. 1. The evolution equations for fragmentation.

with $Q^{2}$ is determined by certain "branching probabilities" $P_{A_{\sigma} B_{\sigma^{\prime}}}$ for parton $B$, helicity $\sigma$ to split into parton $A$, helicity $\sigma^{\prime}$. (Flavor independence of $S U_{3^{-}}$ color interactions makes the splitting functions for quarks flavor independent.) The "evolution equations" are easier to draw than to describe and are depicted in Fig. 1. The corresponding formulae are ${ }^{[3][6]}$

$$
\begin{aligned}
& \frac{d}{d t} D_{q_{\sigma}}^{h_{\lambda}}=\frac{\alpha_{s}}{2 \pi}\left[P_{q q} \otimes D_{q_{\sigma}}^{h_{\lambda}}+P_{G_{\sigma^{\prime}} q_{\sigma}} \otimes D_{G_{\sigma^{\prime}}}^{h_{\lambda}}\right] \\
& \frac{d}{d t} D_{G_{\sigma}}^{h_{\lambda}}=\frac{\alpha_{s}}{2 \pi}\left[P_{q_{\sigma^{\prime}} G_{\sigma}} \otimes D_{q_{\sigma^{\prime}}}^{h_{\lambda}}+P_{G_{\sigma^{\prime}} q_{\sigma}} \otimes D_{G_{\sigma^{\prime}}}^{h_{\lambda}}\right]
\end{aligned}
$$

where $t \equiv \ln Q^{2}$ and $\otimes$ denotes convolution. ${ }^{[2]}$ We have taken advantage of the helicity conservation of the vector coupling to define the quark $P_{q_{\sigma} q_{\sigma}^{\prime}} \equiv P_{q q} \delta_{\sigma \sigma^{\prime}}$. (Parity conservation in strong interactions implies that it is the same for $\sigma= \pm$.) We observe that the evolution equations for the fragmentation functions are simply the transpose of the corresponding equations ${ }^{[1]}$ for structure functions. Because of parity invariance, the equations simplify if we define sum and difference fragmentation functions. The sum

$$
D_{A}^{h_{\lambda}} \equiv D_{A_{+}}^{h_{\lambda}}+D_{A_{-}}^{h_{\lambda}},
$$


may, by parity invariance, also be expressed as $D_{A_{+}}^{h_{\lambda}}+D_{A_{+}}^{h_{-\lambda}}$. The asymmetry for partons of opposite helicity is given by the corresponding differences

$$
\Delta D_{A}^{h_{\lambda}} \equiv \Delta D_{A_{+}}^{h_{\lambda}}-\Delta D_{A_{-}}^{h_{\lambda}}=\Delta D_{A_{+}}^{h_{\lambda}}-\Delta D_{A_{+}}^{h_{-\lambda}}
$$

The unpolarized fragmentation functions are defined as $D_{A}^{h} \equiv \sum_{\lambda} D_{A_{+}}^{h_{\lambda}}$. For quarks, the unpolarized "singlet" and "nonsinglet" fragmentations are defined as $^{\star}$

$$
\begin{aligned}
& D_{S}^{h} \equiv \sum_{\text {flavor }} D_{q_{f}}^{h}+D_{q_{f}}^{h} \\
& D_{N S}^{h} \equiv D_{q_{f}}^{h}-\frac{1}{2 f} D_{S}^{h}
\end{aligned}
$$

Analogously, nonsinglet fragmentations may be formed for the sum $D_{N S}^{h_{\lambda}}$ and asymmetric $\Delta D_{N S}^{h_{\lambda}}$ fragmentations. All obey the same simple evolution equation

$$
\frac{d}{d t} D_{N S}^{h_{\lambda}}=\frac{\alpha_{s}}{2 \pi} D_{N S}^{h_{\lambda}} \otimes P_{q q}
$$

Just as for singlet structure functions, the singlet fragmentations remain coupled and evolve according to

$$
\begin{aligned}
& \frac{d}{d t} \Delta D_{q}^{h_{\lambda}}=\frac{\alpha_{s}}{2 \pi}\left[\Delta P_{q q} \otimes \Delta D_{q}^{h_{\lambda}}+\Delta P_{G q} \otimes \Delta D_{G}^{h_{\lambda}}\right] \\
& \frac{d}{d t} \Delta D_{G}^{h_{\lambda}}=\frac{\alpha_{s}}{2 \pi}\left[\Delta P_{q G} \otimes \Delta D_{q}^{h_{\lambda}}+\Delta P_{G G} \otimes \Delta D_{G}^{h_{\lambda}}\right] .
\end{aligned}
$$

CLEO has recently published ${ }^{[1]}$ their data acquired in $e^{-} e^{+}$annihilation at $10.55 \mathrm{GeV}$ for the unpolarized fragmentation function $D_{c}^{D^{*}}$, Fig. 2, in which ARGUS data ${ }^{[13]}$ at about the same $Q^{2}$ is also plotted. The curve is a phenomenological model by a Lund group ${ }^{[21]}$ which works very well. In comparing their best fit to this data with a similar distribution obtained by HRS in $e^{-} e^{+}$ annihilation at $30.4 \mathrm{GeV}$, it is important to take into account the evolution of this distribution, as shown in Fig. $3 .^{[1]}$ There is no data as yet on the polarized fragmentation functions, but we can be certain that at SLC and possibly LEP, for example, when such data become available at much higher $Q^{2}$, it will be

* For the purposes of studying evolution, the flavor index $f$ may be suppressed in the nonsinglet fragmentation function. Also, there are alternate, sometimes phenomenologically more useful, definitions of the nonsinglet function possible, for example, $D_{N S}^{h} \equiv$ $D_{q_{f}}^{h}-D_{\bar{q}_{f}}^{h}$, obeying the same evolution equation. 
even more important to take into account nonscaling effects predicted by QCD when comparing data.
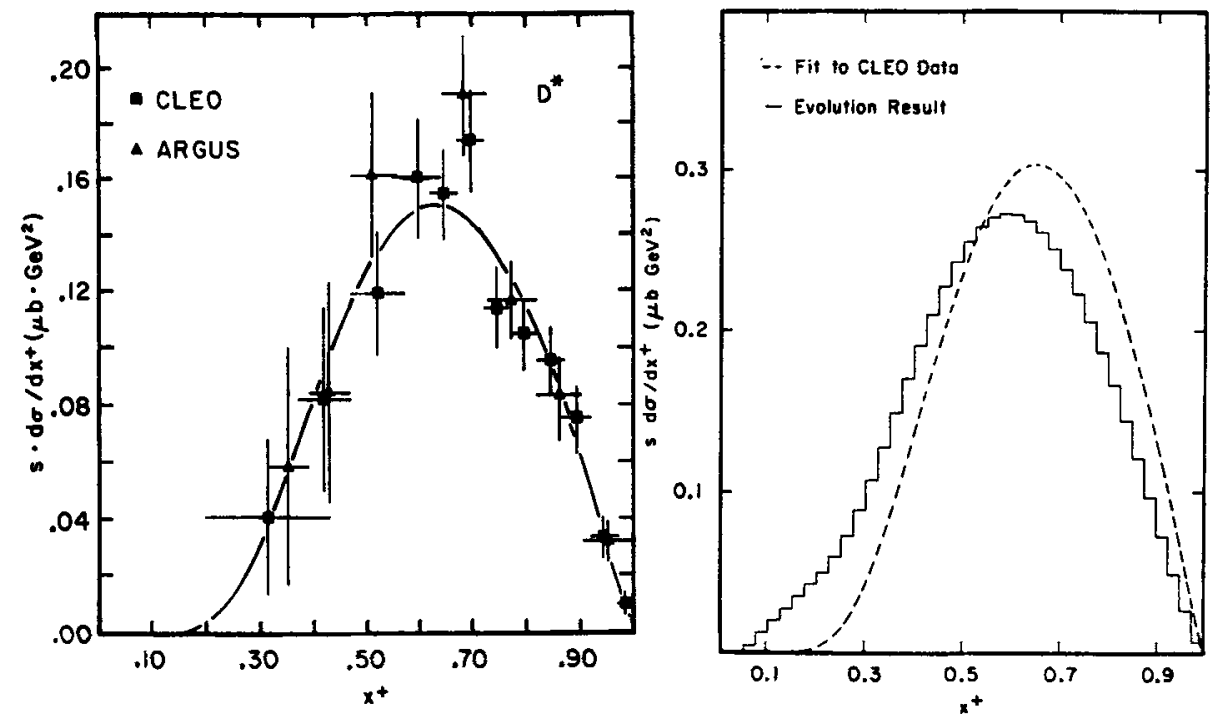

Fig. 2. The unpolarized fragmenta- Fig. 3. The evolution of $c \rightarrow D^{*}$ from tion function for $c \rightarrow D^{*+}$. (Fig. $15,10.5 \mathrm{GeV}$ to $30.4 \mathrm{GeV}$. (Fig. 16, from from ref. 11). ref. 11).

These convolutions are reduced to algebraic equations by forming moments in the usual way

$$
<\Delta D_{N S}^{h_{\lambda}}>_{n} \equiv \int_{0}^{1} d z z^{n-1} \Delta D_{N S}^{h_{\lambda}},
$$

and similarly for the other fragmentation functions. We defer a discussion of the general features to our lengthier publication. ${ }^{[3]}$ Here we will simply note that $<\Delta D_{N S}^{h_{\lambda}}>_{1}$ is independent of $Q^{2}$. On the other hand, both the quark singlet and gluon fragmentation asymmetries grow with $Q^{2}{ }^{[8][8]}$

$$
\begin{gathered}
<\Delta D_{G}^{h_{\lambda}}>_{1}=<\Delta D_{G}^{h_{\lambda}}>_{1}^{0} \frac{t}{t_{0}} \\
<\Delta D_{q}^{h_{\lambda}}>_{1}=<\Delta D_{q}^{h_{\lambda}}>_{1}^{0}+\frac{12}{33-2 f}<\Delta D_{G}^{h_{\lambda}}>_{1}^{0}\left(\frac{t}{t_{0}}-1\right),
\end{gathered}
$$

where $t \equiv \ln Q^{2}$. This is analogous to the logarithmic growth of the mean gluon spin asymmetry in structure functions but differs from the constancy of the mean quark spin asymmetry. ${ }^{[8][0]}$ In particular, this implies that $\alpha_{s}\left\langle\Delta D_{G}^{h_{\lambda}}\right\rangle_{1}$ and $\alpha_{s}<\Delta D_{q}^{h_{\lambda}}>_{1}$ are really of $O(1)$ and not of $O\left(\alpha_{s}\right)$, just as $\alpha_{s} \Delta G$ is $O(1)$ for hadronic structure functions. 
In conclusion, the subject of spin correlations in quark and gluon fragmentation have hardly been thought about. Yet it may turn out that there are extremely large correlations which could become especially useful if new and unusual phenomena ever appear in collisions at very high energies.

\section{REFERENCES}

1. G. Altarelli and G. Parisi, Nucl. Phys. B126 (1977) 298.

2. For a review see G. Altarelli, Phys. Rep. 81 (1982) 1.

3. J. F. Owens, Phys. Lett. 76B (1978) 85; T. Uematsu, Phys. Lett. 79B (1978) 97.

4. M. B. Einhorn in Proceedings of the Workshop on Polarized Beams at the SSC, A. Krisch et.al. (eds.), AIP Conference Proceedings No. 145; NY: AIP, 1986, pp. 149-153.

5. M. B. Einhorn, in preparation.

6. C. S. Lam et.al., Phys. Lett. 106B (1981) 411.

7. C. S. Lam and B.-A. Li, Phys. Rev. D25 (1982) 683.

8. E. N. Argyres, C. S. Lam, and Bing-An Li, Zeit. Phyz. C17 (335) 1983.

9. M. B. Einhorn and J. Soffer, Nucl. Phys. B274 (1986) 714.

10. For a review, see B. Andersson et.al., Phys. Rep. 97 (1983) 31.

11. CLEO Collaboration, D. Bortoletto et.al. Phys. Rev. D37 (1988) 1719, and earlier references therein.

12. HRS Collaboration, M. Derrick et.al., Phys. Lett. 146B (1994) 261; Phys. Rev. Lett. 53 (1984) 522.

13. ARGUS Collaboration, H. Albrecht et.al., Phys. Lett. 153B (1985) 343, 150B (1985) 235.

14. HRS Collaboration, S. Abachi et al., Phys. Lett. 199B (1987) 585.

15. See for example the lectures by J. Dorfan in the Theoretical Advanced Study Institute. Lectures in Elementary Particle PHysics, 1984 D.N. Williams (ed.) Ann Arbor, 1984.

16. R. H. Dalitz, G. R. Goldstein, and R. Marshall, Oxford U. OXFORDTP-70/88 (Oct. 1988). For further discussion by the same authors of the possible correlations of the $c$-quark helicity with the $D \pi$ decay plane, see Oxford U. preprint, Nov., 1988, (submitted to Z. Phys. C.)

17. H.A. Olsen, P. Osland, I. Øverbø, Nucl. Phys. B192 (1981) 33; B171 (1980) 209.

18. See the contributions to this volume by R. D. Carlitz and E. Leader. 
19. See the discussion and references in C. Bourrely, J. Soffer, and E. Leader, Phys. Rep. 59 (198) 95.

20. See the contribution to these Proceedings by A. Yokosawa et.al.

21. B. Andersson, G. Gustafson, and B. Soderberg, Zeit. Phyz. C20 (1983) 317; see also B. Andersson et.al. Phys. Rep. 97 (1983) 33. 